\title{
The effect of xerostomia and hyposalivation on the quality of life of patients with type II diabetes mellitus
}

\author{
Tahereh Molania ${ }^{1}$, Mona Alimohammadi ${ }^{2}$, Ozra Akha $^{3}$, Jaber Mousavi $^{4}$, Ramin Razvini $^{5}$, Maedeh Salehi ${ }^{1}$
}

${ }^{1}$ DDS, MS of Oral and Maxillofacial Medicine, Assistant Professor, Department of Oral Medicine, Faculty of Dentistry, Mazandaran University of Medical Sciences, Sari, Iran

${ }^{2}$ DDS, MS of Oral and Maxilofacial Radiology, Assistant Professor, Department of Oral and Maxilofacial Radiology, Faculty of Dentistry, Mazandaran University of Medical Sciences, Sari, Iran

${ }^{3} \mathrm{MD}$, Endocrinologist, Associate Professor, Diabetes Research Center, Mazandaran University of Medical Sceinse, Sari, Iran

${ }^{4}$ MD, MPH, Board of Community \& Preventive Medicine, Assistant Professor, Department of Community \& Preventive Medicine, Faculty of Medicine, Mazandaran University of Medical Sciences, Sari, Iran

${ }^{5}$ DDS, Sari, Mazandaran, Iran

\section{Type of article: Original}

\begin{abstract}
Background: Diabetes mellitus is a chronic metabolic disease which can have numerous physical effects for patient. Xerostomia is one of these complications. Compared to healthy people, patients with diabetes mellitus, have a worse quality of life, and complications of diabetes are the main determinants of quality of life in these patients.

Objective: The aim of this study was to determine the effects of xerostomia and hyposalivation on quality of life of patients with type 2 diabetes mellitus.

Methods: This descriptive-analytical epidemiological study was conducted on 200 patients with type 2 diabetes mellitus referred to the diabetes clinic of Shahid Mostafavi in Sari city from October 2015 to January in 2016. A questionnaire containing personal characteristics and medical situation was completed by each person. Then, the Persian Oral Health Impact Profile-14 (OHIP-14-PER) questionnaire was completed by the patients. Eventually, with the use of chewable paraffin for $1.5 \mathrm{~min}$ by the patient, stimulated salivary flow rate (SSFR) test was performed, and in order to determine hyposalivation, their saliva amount underwent a gravimetric test. Finally, using statistical software SPSS16, the information was statistically analyzed by independent-samples t-test, Mann-Whitney U, Chi-squared and fisher exact tests.

Results: The average age of patient was 56.41 years old ( $43 \%$ male and $57 \%$ female). Mean SSFR was 0.7 $\mathrm{ml} / \mathrm{min}$ in patients and xerostomia were confirmed in 112 patients. Difference between age, gender, drug use, years affecting to diabetes and FBS amount in patient with hyposalivation were not statistically meaningful in proportion to patients without it. But difference between HbA1C and SSFR in patients with hyposalivation were statistically meaningful than to patients without it $(\mathrm{p}=0.03, \mathrm{p}=0.001$ respectively). The mean patient score to OHIP-14 were obtained as 38.17 . The questionnaire score difference in patients with hyposalivation in proportion to patients without it were not statistically meaningful.

Conclusion: Hyposalivation possibility increases in diabetic patients with low metabolic control which can cause more severe side effects in relation to oral health. Xerostomia in diabetic patients has negative effects on oral health related quality of life. Diabetic control and patients' oral problem improvement is effective in their quality of life promotion.
\end{abstract}

Keywords: Diabetes mellitus, Xerostomia, Saliva

\section{Corresponding author:}

Assistant Professor Dr. Maedeh Salehi, Department of Oral Medicine, Faculty of Dentistry, Mazandaran University of Medical Sciences, Sari, Iran. Tel: +98.1133244894, Email: salehimaedeh1165@gmail.com

Received: May 03, 2017, Accepted: September 24, 2017, Published: November 2017

iThenticate screening: September 18, 2017, English editing: October 14, 2017, Quality control: November 15, 2017 This article has been reviewed / commented by three experts

(C) 2017 The Authors. This is an open access article under the terms of the Creative Commons Attribution-NonCommercialNoDerivs License, which permits use and distribution in any medium, provided the original work is properly cited, the use is non-commercial and no modifications or adaptations are made. 


\section{Introduction}

Diabetes mellitus is a chronic metabolic disease that can have physical effects on patients. Xerostomia (dry mouth sensation) is one of these complications. Reduction in salivary flow resulting in xerostomia leads to speaking and chewing problems, mucosal inflammation, candidal infections and atrophic changes of oral mucosa, increase of plaque accumulation, decrease of buffering capacity of the saliva and increase of decay (1). Many studies have examined the prevalence of oral lesions and xerostomia in patients with diabetes. In one study, the prevalence of dry mouth in patients with diabetes was a reported $76.4 \%$ (1). In addition, it was found that this complication could have negative effects on the quality of life, so the xerostomia should be diagnosed correctly in this group of patients to treat its negative effects on oral health and to achieve a higher quality of life (2). Xerostomia is the subjective sensation of dry mouth and this definition is based on the presence or absence of individual feeling of dry mouth and not based on actual measurements of salivary flow rate, while hyposalivation is defined as the objective symptoms of a reduced salivary flow rate. Subjective xerostomia can be related to hyposalivation and other physiological factors. Changes in salivary composition instead of changes in salivary quantity may produce xerostomia (3). Narhi reported that dry mouth sensation was not necessarily related with decreased salivary flow and it could be seen in people with normal salivary flow. The prevalence of xerostomia and hyposalivation was reported to be approximately $20 \%$, but the simultaneous existence of both was only $6 \%$ (4). The term "quality of life" (QOL) is defined as the patients' feeling of well-being related to various aspects of life. Oral health related quality of life (OHRQoL) consists of individual feelings of the effects of oral health on the quality of personal life. Patients with diabetes mellitus, compared to healthy people, have a worse quality of life, and complications of diabetes are the main determinants of quality of life in these patients (5). In 1997, Slide changed the Oral Health Impact Profile-49 (OHIP-4) questionnaire into the new and short-form version named OHIP-14 questionnaire by maintaining its validity and reliability to evaluate the clinical situation and its effect on the QOL for patients. After that, OHIP-14 questionnaire has been used to evaluate the effects of oral health on the QOL (5). Recent research has examined the relationship between xerostomia and QOL (6-8). In a study that was conducted in 2014, they examined oral health related to quality of life in patients with type 2 diabetes mellitus and found that oral health problems in diabetic patients depend on medical conditions and levels of blood glucose control of which these problems have a negative effect on their quality of life (9). Considering the high prevalence of diabetes among the population and the high percentage of oral complications of disease, particularly xerostomia, the aim of this study was to evaluate the effect of xerostomia and hyposalivation on the QOL of patients with type 2 diabetes mellitus. And finally, the quality of life questionnaire was compared in diabetic patients with xerostomia and hyposalivation.

\section{Material and Methods}

This descriptive-analytical epidemiological study was conducted on 200 patients with type 2 diabetes mellitus referred to the diabetes clinic of Shahid Mostafavi in Sari city from October 2015 to January in 2016. Inclusion criterion was patients with type 2 diabetes with a dry mouth. Exclusion criteria were illiterate patients or those who did not have the ability to fill out the questionnaire and also those who had systemic conditions such as hypothyroidism, history of radiotherapy in head and neck area or chemotherapy during the past 3 months or consumers of drugs and psychotropic substances, smokers, alcoholics and consumers of medications which cause dry mouth (antihistamines, anticholinergics, anti-hypertensive, tricyclic antidepressants), respectively (10). At first, the plans and aims of the study were explained to the participants, and oral consent was obtained from all patients. Demographic information including age, gender, duration of diabetes, type of medications was written in their records. Fast blood sugar (FBS) level during the study and Hemoglobin A1c (HbA1C) level in less than three months before the study were also recorded (10). Patients with $\mathrm{HbA} 1 \mathrm{C} \leq 8 \%$ were as persons with good control and patients with $\mathrm{HbA} 1 \mathrm{c}>8 \%$ were as uncontrolled ones. Moreover, FBS level ranged from 90 to $130 \mathrm{mg} / \mathrm{dl}$ was defined as control of good blood sugar (10).

\subsection{Xerostomia and hyposalivation assessment}

To determine the presence of xerostomia, each patient was asked about dry mouth during the day over the last 6 months and a positive response indicated dry mouth in patients, and after that, three additional questions were asked: 1- How would you describe the saliva in your mouth? 2- Do you have trouble in swallowing? 3- Do you need a drink to swallow food? Then their answers to these questions were recorded (10). Hyposalivation was examined via collecting the stimulated saliva. All sampling was conducted in the period from 9 to 11 am. The subjects were asked to avoid smoking, eating food and drinking water at least 2 hours before sampling. Also, patients with partial and complete dentures were asked to remove them before testing the stimulated saliva, and to wash their mouth for 1 minute before sampling, and then their oral cavity was evaluated to ensure no possibility of substances were in their oral cavity. Patients were seated on a chair when swallowing their saliva. Then, $1 \times 1 \mathrm{~cm}$ paraffin manufactured by 
Van (Iran) was put in their mouth and sucked for 1.5 minutes. Afterwards, they spat the saliva into a test tube for 3 minutes (11). Next, the saliva samples were kept in a freezer with $-70{ }^{\circ} \mathrm{C}$ temperature until their measuring time (11). Stimulated salivary flow rate (SSFR) was evaluated using the gravimetric method, and expressed in milliliters per minute $(\mathrm{ml} / \mathrm{min})$. SSFR $>0.7 \mathrm{ml} / \mathrm{min}$ showed the normal salivary flow and SSFR $\leq 0.7 \mathrm{ml} / \mathrm{min}$ indicated the hyposalivation (10).

\subsection{Assessment the effect of xerostomia on quality of life}

OHIP-14-Per questionnaire whose validity and reliability had been confirmed by Motalleb Nejad et al. was used to evaluate the effect of xerostomia on QOL in patients (12). The patients were asked to respond to the questions regarding the period of the past 6 months. The questionnaire contained 14 multiple-choice questions with five choices for each question and the five choices with their scores are as follows: Never $=1$, seldom $=2$, sometimes $=$ 3 , almost often $=4$, in the majority of cases $=5$. This questionnaire covered seven aspects of quality of life related to oral health including functional limitation, physical pain, psychological discomfort, physical disability, psychological disability, social disability and handicap. All questions were negative in this questionnaire and therefore the scores of all questions had inverse relationship with good oral conditions. Total scores of OHIP-14 per (Additive Score of OHIP = ADD-OHIP) were calculated so that the minimum and maximum scores were 14 and 70, respectively (12). Higher scores indicated the negative impact on QOL. Because some patients might not distinguish among the words "sometimes", "almost often" and "in the majority of cases", the simple count (SC) method was used. It means that, if the patient chose one of two choices (almost often and in the majority of cases), the score of the question would be 1 and if one of three choices (never, seldom and sometimes) was chosen, the score would be Zero and finally the range of all questions was 0 to 14 so that the higher score indicated quality of life related to a lower oral health (12).

\subsection{Data Analysis}

After collecting the questionnaires, data were analyzed using SPSS-16. OHIP-14 questionnaire was analyzed using Kolmogorov-Smirnov Test and Levene's test was used to analyze the homogeneity of variance. In addition, $25^{\text {th }}$ and $75^{\text {th }}$ percentiles were used to describe the data related to the questionnaire. Other tests used in the study included independent-samples t-test, Mann-Whitney, chi-squared and Fisher's exact tests. P-value less than or equal 0.05 was considered significant.

\section{Results}

\subsection{Characteristics of the study population}

Totally, 200 patients with type 2 diabetes mellitus, who had xerostomia over the last 6 months were entered into the current study. The mean age of patients was 56.41 ranged from 22 to 85 years. In total, 85 and 114 patients were male and female, respectively. Mean of diabetes years was 9.75 (for 1-32 years). FBS mean of patients was 128.43 $\mathrm{mg} / \mathrm{dl}(82-210 \mathrm{mg} / \mathrm{dl})$ during the study, and the mean of HbAlC was $7.2 \%(5.1-9.3 \%)$ in patients over the last 3 months. Among them, 108 patients used oral medications to control diabetes, 67 patients took the drugs by injection and 25 cases used the combination of oral and injectable drugs to treat the diabetes. The mean of SSFR also was 0.7 $\mathrm{mg} / \mathrm{dl}$ and hyposalivation was confirmed in 112 patients (56\%) (Table 1).

\subsection{Evaluation of the study variables}

In a total, 112 of 200 patients with type 2 diabetes mellitus, who complained of xerostomia had hyposalivation after gravimetric measuring of stimulated saliva, and the mean of SSFR was 0.58 in patients with hyposalivation, which suggested that there was a significant relationship between hyposalivation and xerostomia in this study $(\mathrm{p}=0.001)$. When hyposalivation as an independent variable compared to its dependent variables was studied, it was found that the difference of age, gender, medication use, years of diabetes and FBS level in patients with hyposalivation was not statistically significant compared to patients without it. However, the maximum level of FBS was $210 \mathrm{~mL} / \mathrm{min}$ in the current study, observed in diabetics with hyposalivation and patients without hyposalivation, had better control on their FBS. Also in this study, the maximum year of affecting diabetes was 32, which was reported in patients with hyposalivation. However, there was statistically significant difference between the two variables including SSFR and HbA1C in patients with hyposalivation than without hyposalivation ( $\mathrm{p}=0.001$ and 0.03 respectively). In studying of qualitative variables including three questions on the flow rate of saliva, difficulty in swallowing and need to drink liquids while eating, interesting results were obtained in the present study. In all the above questions, the difference in answers of patients with hyposalivation compared to without hyposalivation was statistically significant $(\mathrm{p}<0.001)$ (Table 2). 
Table 1. Characteristics of the studied population

\begin{tabular}{|l|l|l|l|}
\hline \multicolumn{2}{|l|}{ Variable } & n & mean \pm SD or $\%$ \\
\hline Age (year) & 200 & $56.41 \pm 13.1$ \\
\hline \multirow{2}{*}{ Gender } & Female & 114 & 43 \\
\cline { 3 - 4 } & Male & 86 & 57 \\
\hline Time with DM 2 (year) & 200 & $9.75 \pm 5.1$ \\
\hline FBS (Mg/dl) & 200 & $128.43 \pm 17.2$ \\
\hline HBA $_{1} \mathrm{C}(\%)$ & 200 & $7.24 \pm 0.8$ \\
\hline ADD-oHIP-14 & 200 & $38.17 \pm 7.8$ \\
\hline SC-oHIP-14 & 200 & $11.25 \pm 3.1$ \\
\hline SSFR (Ml/min) & & 200 & $0.7 \pm 0.1$ \\
\hline Amount of saliva referred & Low & 137 & 68.5 \\
\cline { 2 - 4 } & Normal & 63 & 31.5 \\
\hline \multirow{2}{*}{ Difficulty in swallowing } & Yes & 94 & 48 \\
\cline { 2 - 4 } & No & 104 & 52 \\
\hline Need to drink & Yes & 72 & 36 \\
\cline { 2 - 4 } & No & 128 & 64 \\
\hline \multirow{2}{*}{ Hyposalivation } & Yes (SSFR $\leq 0.7(\mathrm{ml} / \mathrm{min}))$ & 112 & $0.58 \pm 0.1$ or 56 \\
\cline { 2 - 4 } & No (SSFR $>0.7(\mathrm{ml} / \mathrm{min}))$ & 88 & $0.86 \pm 0.09$ or 44 \\
\hline
\end{tabular}

Table 2. Analysis of hyposalivation as a dependent variable showing the independent variable in terms of mean \pm $\mathrm{SD}$ or number of occurrences and odds ratio (OR) and $\mathrm{p}$-value

\begin{tabular}{|c|c|c|c|c|c|c|}
\hline \multirow{2}{*}{\multicolumn{2}{|c|}{ Independent variable }} & \multicolumn{5}{|c|}{ Dependent variable } \\
\hline & & Yes & NO & $\mathrm{N}$ & OR & p-value \\
\hline \multicolumn{2}{|l|}{ Age (year); Mean \pm SD } & $56.64 \pm 14.2$ & $56.13 \pm 11.5$ & 200 & - & 0.77 \\
\hline \multirow[t]{2}{*}{ Gender (\%) } & Female & 30.5 & 26.5 & 112 & \multirow[t]{2}{*}{1.26} & \multirow[t]{2}{*}{0.41} \\
\hline & Male & 25.5 & 17.5 & 88 & & \\
\hline \multicolumn{2}{|c|}{ Time with $\mathrm{DM}_{2}$ (year) $;$ Mean \pm SD } & $10.44 \pm 6.4$ & $8.89 \pm 5.2$ & 200 & - & 0.06 \\
\hline \multicolumn{2}{|c|}{ FBS $(\mathrm{Mg} / \mathrm{dl}) ;$ Mean \pm SD } & $130.21 \pm 18.8$ & $126.16 \pm 14.9$ & 200 & - & 0.1 \\
\hline \multicolumn{2}{|l|}{$\mathrm{HBA}_{1} \mathrm{C}(\%) ;$ Mean $\pm \mathrm{SD}$} & $7.35 \pm 0.7$ & $7.1 \pm 0.8$ & 200 & - & $0.03^{*}$ \\
\hline \multicolumn{2}{|l|}{ ADD-oHIP-14; Mean \pm SD } & $38.38 \pm 9.3$ & $37.9 \pm 9.3$ & 200 & - & 0.67 \\
\hline \multicolumn{2}{|l|}{ SC-oHIP-14; Mean \pm SD } & $11.25 \pm 2.8$ & $11.25 \pm 3.5$ & 200 & - & 1 \\
\hline \multirow[t]{3}{*}{ Medication } & Oral & 54 & 54 & 108 & \multirow[t]{3}{*}{ - } & \multirow[t]{3}{*}{0.17} \\
\hline & Injection & 42 & 25 & 67 & & \\
\hline & Combination & 16 & 9 & 25 & & \\
\hline \multicolumn{2}{|l|}{ SSFR (Ml/min) ; Mean \pm SD } & $0.58 \pm 0.1$ & $0.86 \pm 0.9$ & 200 & - & $0.001^{*}$ \\
\hline \multirow[t]{2}{*}{ Perceived amount of saliva } & Low & 93 & 44 & 137 & \multirow[t]{2}{*}{4.89} & \multirow[t]{2}{*}{$0.001^{*}$} \\
\hline & Normal & 19 & 44 & 63 & & \\
\hline \multirow[t]{2}{*}{ Difficulty in swallowing } & Yes & 66 & 30 & 96 & \multirow[t]{2}{*}{2.94} & \multirow[t]{2}{*}{$0.001^{*}$} \\
\hline & No & 58 & 46 & 104 & & \\
\hline \multirow[t]{2}{*}{ Need to drink } & Yes & 60 & 20 & 72 & \multirow[t]{2}{*}{2.77} & \multirow[t]{2}{*}{$0.001^{*}$} \\
\hline & No & 52 & 68 & 128 & & \\
\hline
\end{tabular}

\subsection{Study of the effect of xerostomia and hyposalivation on quality of life}

Regarding the study of the OHRQoL, a total of 200 patients responded to all questions. Average score of ADD OHIP - 14 with a range of (21 to 67) and SC - OHIP - 14 with a range of (0 to 14) was $38.17 \pm 7.84$ and $11.25 \pm 3.18$, respectively. There was no significant difference between patients with hyposalivation and without it in terms of ADD - OHIP - 14 via statistical analysis using independent-samples t-test. The same results were obtained in relation to SC -OHIP - 14 and the difference was not significant either. OHIP - 14 questionnaire showed that patients had more problem in the field of physical pain so that $58 \%$ of patients chose the choices of "almost often" and "in the majority of cases" for two questions concerning this aspect. 


\section{Discussion}

Dry mouth is as an oral manifestation of diabetes that can lead to many clinical or social problems for patients (1). In the present study, diagnosis of xerostomia was confirmed based on the feeling of dry mouth in patients over the last 6 months, then this assessment was completed by three additional questions. Xerostomia is the subjective sensation of dry mouth and can occur in people with normal salivary flow rate. Some believe that saliva compounds can be more effective than its flow in causing xerostomia. However, hyposalivation is the actual reduction in salivary flow rate so the SSFR and unstimulated salivary flow rate are used to diagnose it (3). Unstimulated salivary flow rate is influenced more than a stimulated one by hours of the day, the patient's physical and psychological state. In addition, its collection due to its low flow rate is longer (7). Therefore, in the present study, SSFR was measured to estimate the relationship between hyposalivation and xerostomia. Busato et al. studied 51 patients with type 1 diabetes and they reported that the prevalence of xerostomia was 52.9\% although there was no relationship between hyposalivation and xerostomia in their study (10). In other studies, there was relationship between hyposalivation and xerostomia in patients with type 1 diabetes mellitus, and some researchers stated that type 1 diabetes was not as a predisposing factor for hyposalivation (13). Unlike them, Chavez et al. reported that diabetics are more vulnerable to hyposalivation than others (14). The prevalence of xerostomia in patients with type 2 diabetes was reported as $76.4 \%$ which was higher than that in patients with diabetes type 1 (10). It seems that hyposalivation due to a higher prevalence of xerostomia is higher in these patients. Hyposalivation in diabetics can lead to many side effects in the oral mucous membrane such as dryness, pain, atrophy, mucositis, ulceration and opportunistic infections (fungal, bacterial and viral), depapillation of tongue and increase of the dental caries (10). In the present study, the difference in the flow of saliva, difficulty in swallowing and the need to drink while eating was higher in patients with hyposalivation than without hyposalivation, and this difference was statistically significant. This represents a real reduction in salivary flow rate and subsequent problems in the swallowing function of patients and a real need to drink in order to facilitate swallowing. The number of affected years of diabetes and its metabolic control can also play a role in creating hyposalivation and xerostomia (10). In the current study, although no significant difference was found between the number of affected years of diabetes and hyposalivation, hyposalivation was observed in people with the maximum years of diabetes. Moreover, the mean years of diabetes and FBS were higher in patients with hyposalivation than without it. Statistically, there was a significant difference between HbA1C and hyposalivation ( $\mathrm{p}=0.03$ ), so that its mean was $7.35 \%$ in people with Hyposalivation. Given that $\mathrm{HbA} 1 \mathrm{C}$ is a good indicator of control and long-term follow-up of diabetes, its higher concentration in hyposalivation group indicates the importance of this indicator in the control of metabolic status of patients with diabetes. In this study, the mean score of ADD - OHIP - 14 was 38.17 which represented the negative effect of xerostomia on QOL of diabetics and this effect was found in other studies by using OHIP - 14 and other questionnaires. In order to increase the accuracy of questionnaires and responses of patients, another method was used to score the OHIP - 14 questionnaires, named $\mathrm{SC}$ - OHIP - 14. The obtained mean of SC - OHIP - 14 was 11.25. There was no significant difference between the two scoring systems when the patients with hyposalivation were compared to those without it. Moreover, its justification is that any systemic condition such as diabetes mellitus or chronic use of multiple medications can affect oral health $(10,15)$. All patients participating in the study were suffering from xerostomia. Therefore, their responses to a questionnaire were based on the subjective sensation of dry mouth and its complications, and there was no difference between the questionnaires of patients with xerostomia and those with hyposalivation.

\section{Conclusions}

Finally, it can be concluded that the possibility of hyposalivation in diabetic patients with poor metabolic control or high number of affected years increases, which can create more serious complications associated with their oral health. Xerostomia in patients with diabetes has a negative effect on quality related to oral health. Further studies are needed to evaluate the association between diabetes control levels and xerostomia leading to hyposalivation in diabetic patients.

\section{Acknowledgments:}

We would like to express our sincere thanks to Mazandaran University of Medical Sciences which approved and sponsored this study with code 1712. We also thank our colleagues in the clinic of Shahid Mostafavi in Sari city.

\section{Conflict of Interest:}

There is no conflict of interest to be declared.

\section{Authors' contributions:}

All authors contributed to this project and article equally. All authors read and approved the final manuscript. 


\section{References:}

1) Mauri-Obradors E, Estrugo-Devesa A, Jané-Salas E, Viñas M, López-López J. Oral manifestations of Diabetes Mellitus. A systematic review. Med Oral Patol Oral Cir Bucal. 2017; 22(5): e586-94. doi: 10.4317/medoral.21655. PMID: 28809366, PMCID: PMC5694181.

2) Carda C, Mosquera-Lloreda N, Salom L, Gomez de Ferraris ME, Peydró A. Structural and functional salivary disorders in type 2diabetic patients. Med Oral Patol Oral Cir Bucal. 2006; 11(4): E309-14. PMID: 16816810.

3) Busato IM, Ignácio SA, Brancher JA, Moysés ST, Azevedo-Alanis LR. Impact of clinical status and salivary conditions on xerostomia and oral health-related quality of life of adolescents with type 1 diabetes mellitus. Community Dent Oral Epidemiol. 2012; 40(1): 62-9. doi: 10.1111/j.1600-0528.2011.00635.x. PMID: 21883355.

4) Narhi TO. Prevalence of subjective feelings of dry mouth in the elderly. J Dent Res. 1994; 73(1): 20-5. doi: 10.1177/00220345940730010301. PMID: 8294614.

5) López-Jornet $\mathrm{P}$, Camacho-Alonso F, Lucero Berdugo M. Measuring the impact of oral mucosa disease on quality of life. Eur J Dermatol. 2009; 19(6): 603-6. doi: 10.1684/ejd.2009.0762. PMID: 19592326.

6) Baker SR, Pankhurst CL, Robinson PG. Utility of two oral health-related quality-of-life measures in patients with xerostomia. Community Dent Oral Epidemiol. 2006; 34(5): 351-62. doi: 10.1111/j.16000528.2006.00285.x. PMID: 16948674.

7) Ikebe $\mathrm{K}$, Matsuda $\mathrm{K}$, Morii $\mathrm{K}$, Wada $M$, Hazeyama $T$, Nokubi $T$, et al. Impact of dry mouth and hyposalivation on oral healthrelated quality of life of elderly Japanese. Oral Surg Oral Med Oral Pathol Oral Radiol Endod. 2007; 103(2): 216-22. doi: 10.1016/j.tripleo.2005.12.001. PMID: 17234539.

8) Saini R, Al-Maweri SA, Saini D, Ismail NM, Ismail AR. Oral mucosal lesions in non oral habit diabetic patients and association of diabetes mellitus with oral precancerous lesions. Diabetes Res Clin Pract. 2010; 89(3): 320-6. doi: 10.1016/j.diabres.2010.04.016. PMID: 20488573.

9) Nikbin A, Bayani M, Jenabian N, Khafri S, Motallebnejad M. Oral health-related quality of life in diabetic patients: comparison of the Persian version of Geriatric Oral Health Assessment Index and Oral Health Impact Profile: A descriptive-analytic study. J Diabetes Metab Disord. 2014; 13(1): 32. doi: 10.1186/22516581-13-32. PMID: 24495383, PMCID: PMC4015305.

10) Busato IM, Ignácio SA, Brancher JA, Grégio AM, Machado MA, Azevedo-Alanis LR. Impact of xerostomia on the quality of life of adolescents with type 1 diabetes mellitus. Oral Surg Oral Med Oral Pathol Oral Radiol Endod. 2009; 108(3): 376-82. doi: 10.1016/j.tripleo.2009.05.005. PMID: 19716505.

11) Agha hosseini F, Parsapoor Moghaddam P, Mirzaii Dizgah I. Stimulated whole saliva composition in dental healthy students and menopausal women in 2004. Oral Disease. 2007; 13(3): 320-3.

12) Motallebnejad M, Hadian H, Mehdizadeh S, Hajiahmadi M. Validity and reliability of the Persian version of the oral health impact profile (OHIP)-14. Caspian J Intern Med. 2011; 2(4): 314-20. PMID: 24551438, PMCID: PMC3895829.

13) Siudikiene J, Machiulskiene V, Nyvad B, Tenovuo J, NedzelskieneI. Dental caries and salivary status in children with type 1diabetes mellitus, related to the metabolic control of the disease. Eur J Oral Sci. 2006; 114(1): 8-14. doi: 10.1111/j.1600-0722.2006.00277.x. PMID: 16460335.

14) Chavez EM, Taylor GW, Borrell LN, Ship JA. Salivary function and glycemic control in older persons with diabetes. Oral Surg Oral Med Oral Pathol Oral Radiol Endod. 2000; 89(3): 305-11. doi: 10.1016/S1079-2104(00)70093-X. PMID: 10710454.

15) Busato IM, Igna'cio SA, Brancher JA, Moyse's ST, Azevedo-Alanis LR. Impact of clinical status and salivary conditions on xerostomia and oral health-related quality of life of adolescents with type 1 diabetes mellitus. Community Dent Oral Epidemiol. 2012; 40(1): 62-9. doi: 10.1111/j.1600-0528.2011.00635.x. PMID: 21883355. 